\title{
Tunable filter based on two cascaded photonic liquid crystal fibers
}

\author{
Marzena M. Tefelska, ${ }^{1}$ Sławomir Ertman, ${ }^{1}$ Tomasz R. Woliński, ${ }^{1}$ Roman Dąbrowski, ${ }^{2}$ Paweł Mergo ${ }^{3}$ \\ ${ }^{1}$ Faculty of Physics, Warsaw University of Technology, Koszykowa 75, 00-662 Warsaw, Poland \\ ${ }^{2}$ Military University of Technology, Warsaw, Poland \\ ${ }^{3}$ Maria Curie Sklodowska University, Lublin, Poland
}

Received March 25, 2013; accepted March 28, 2013; published March 31, 2013

\begin{abstract}
This paper describes a tunable filter based on two independently tuned photonic crystal fibers (PCFs) filled with the same liquid crystal. Photonic bang-gaps (PBGs) spectral position of both PCF sections can be controlled with two separate temperature controllers. Overall transmission of the device depends on PBGs formed in both liquid crystal infiltrated PCF sections. As a result, in such a cascaded device both position and bandwidth of the guided waves can be controlled.
\end{abstract}

A typical photonic crystal fiber $[1,2](\mathrm{PCF})$ is an optical fiber in which a micro-periodic dielectric structure is made of silica glass. It consists of a solid core or a hollow core surrounded by a cladding with micro holes arranged in a hexagonal structure. In the last few years there has been an increasing interest of many researchers around the world in photonic crystal fibers (PCFs) [3, 4] and their potential applications.

Generally, in a solid-core PCF light can be guided by the index-guiding mechanism that is similar to a classical waveguide effect based on total internal reflection. The properties of PCFs can be changed by infiltrating their airholes with various substances. Photonic liquid crystal fibers (PLCFs) are PCFs which are infiltrated with liquid crystals (LC). PLCFs whose averaged effective refractive index in the cladding regions is higher than the refractive index of the fiber core, will no longer guide light by the index-guiding mechanism, but become a photonic bandgap (PBG) guiding fiber. In this case, the guiding mechanism relies on the coherent backscattering of light into the core. PBG guiding is usually met in hollow-core photonic crystal fibers [5].

The most interesting substances for PCF infiltration are liquid crystals (LC) which properties can be easily modified by external fields [6-9]. By tuning LCs properties it is possible to tune properties of the fiber and obtain tunable fiber devices. Consequently, several optical devices [10, 11] based on photonic crystal fibers filled with liquid crystal such as: tunable filters [12], phase shifters [13] and optical attenuators [14] have already been demonstrated.

The aim of this study was to build a cascade filter based on a photonic crystal fiber filled with liquid crystals where it was possible to change the width of the output signal by shifting the photonic band gaps position by using temperature control.

The idea of an ideal cascade filter is presented in Fig. 1a, where there are two filters with a rectangular shape effective transmission spectra will be defined by overlap of both spectra. For an ideal filter we assume rectangular transmission with no losses (transmission=1). If two ideal rectangular filters are cascaded, only the common part of their spectrum will be transmitted. Therefore the output signal will be narrowed but the level of transmission will be kept at 1 .
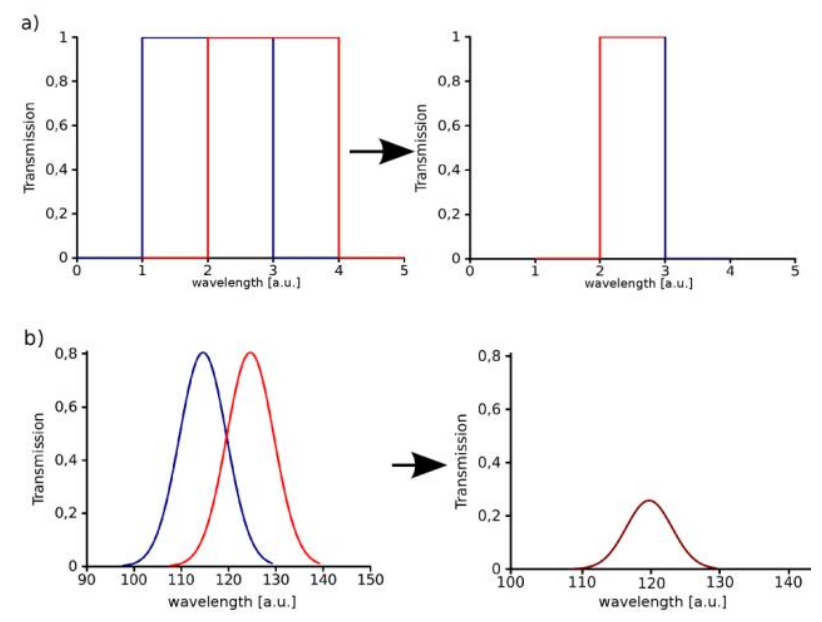

Fig. 1. The idea of a cascade filter: a) an ideal filter with a rectangle signal - transmission is on the same level, b) a non-ideal filter where transmission is reduced.

The shape of transmission spectra of the photonic crystal fiber filled with a liquid crystal is, of course, not rectangular. We decided to use a photonic liquid crystal fiber with one dominant and broad PBG, in which the transmission is about 0.5 . The output signal of two interconnected PLCFs forming the cascade device will be narrowed but their effective transmission level will decrease proportionally (as in Fig. 1b). 
In this experiment two PCFs were filled with the same liquid crystal by capillary action. Then the liquid crystal was pushed into the fiber by using an air compressor.

Both PLCFs were connected together "face to face" (Fig. 2) and placed into a measuring system. The sections filled with LC were placed on Peltier modules.

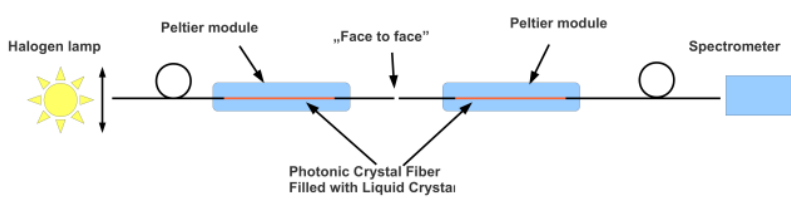

Fig. 2. Experimental setup.

The experimental setup consists of a halogen lamp (Ocean Optics Mikropack) used as a light source and the Ocean Optics HR4000 spectrometer with $0.2 \mathrm{~nm}$ optical resolution that served as a detector. The temperature was changed by the Peltier modules.

In this work, 1023 061215P1 LC19 PCF host-structures (Fig. 3) fabricated at the Maria Curie-Sklodowska University in Lublin (UMCS) were used. The manufactured fiber is characterized by the following parameters: lattice constant (period) $\Lambda=6 \mu \mathrm{m}$, diameter of the holes $\mathrm{d}=3.8 \mu \mathrm{m}, \mathrm{d} / \Lambda=0.63$.

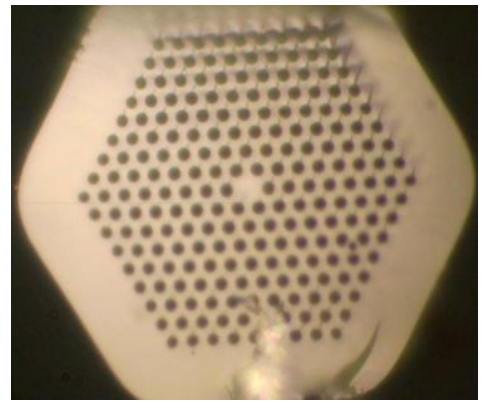

Fig. 3. Cross-section of the photonic crystal fibers with 8 rings of holes (1023)

A low-birefringence 1550 LC mixture, synthesized at the Military University of Technology (Warsaw, Poland) was used for the infiltration. Its refractive indices temperature characteristics are presented in Fig. 4. The details of this mixture are described in [16]. The 1550 LC mixture with 1023 PCF yields a wide photonic band gap on the output signal so it was chosen for cascade filter manufacture. It was very important to obtain two PLCFs with almost identical signals. In our previous works [15, 17] we noticed that the 1023 PCF with the 1550 LC mixture gives highly repeatable wide band gaps with repeatable tuning.
It was the reason for selecting a combination of the 1023 PCF and the 1550 LC to construct the cascade filter.

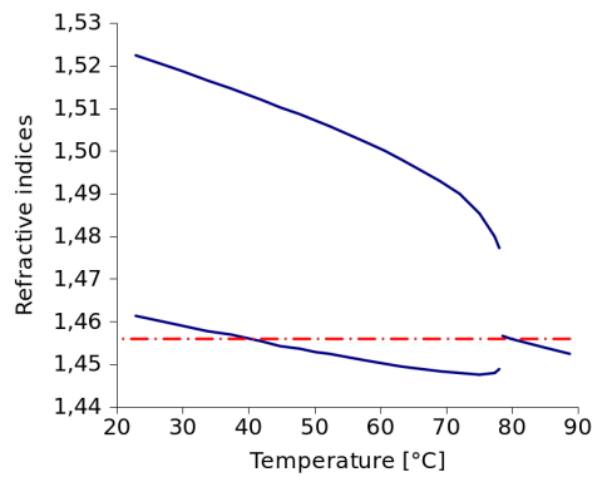

Fig. 4. Thermal characteristic of refractive indices for $1550 \mathrm{LC}$ mixture.

Two sections of the $1023 \mathrm{PCF}$ with a length of $40 \mathrm{~cm}$ were infiltrated with the $1550 \mathrm{LC}$ mixture at a distance of 2.5 $\mathrm{cm}$.

As expected, a decrease in transmission signal was obtained (Fig. 5).

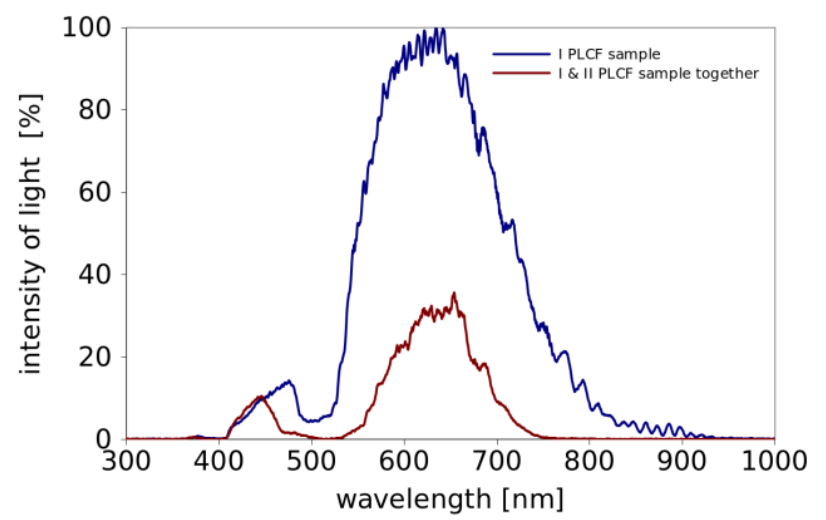

Fig. 5. The output signal of PLCF (blue line) and decreased light intensity after passing through the I \& II PLCFs (brown line).

In Fig. 6 the photonic band gaps are presented. PBG of the first PLCF is marked on the chart by the blue line and the second one is marked by the green line. PBGs are not in the same position and the outline is not exactly the same. This follows from the filling process, fiber structure and a slight change of room temperature while measured. The orange line is the signal after passing through two PLCFs which appeared to be narrowed. 


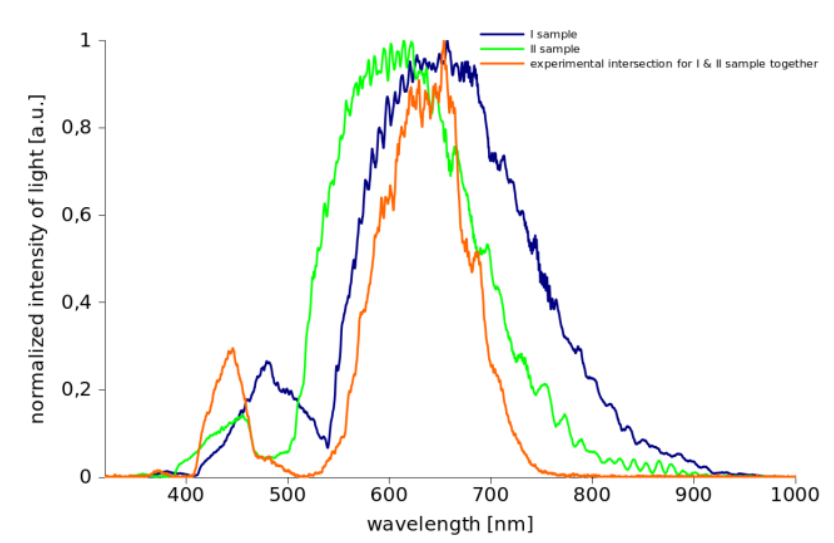

Fig. 6. Experimentally measured photonic band-gaps of two samples (green and blue line). The orange line shows the narrowed band-gap measured at the output of combined samples.

In Fig. 7 the process of narrowing $P B G$ under the influence of temperature is presented. One of the PCFs, filled with LC was heated by a single Peltier module while the other one was kept in stable room temperature. The PBG moved toward shorter wavelengths when the temperature was increasing and, simultaneously, PBG was narrowing. The same effect occurred in the case where the temperature was decreased but in this situation PBGs were moving toward the longer wavelengths. In Figs. 5 and 6 the output signal was normalized in order to describe the shape of the signal and the narrowing effect of PBGs.

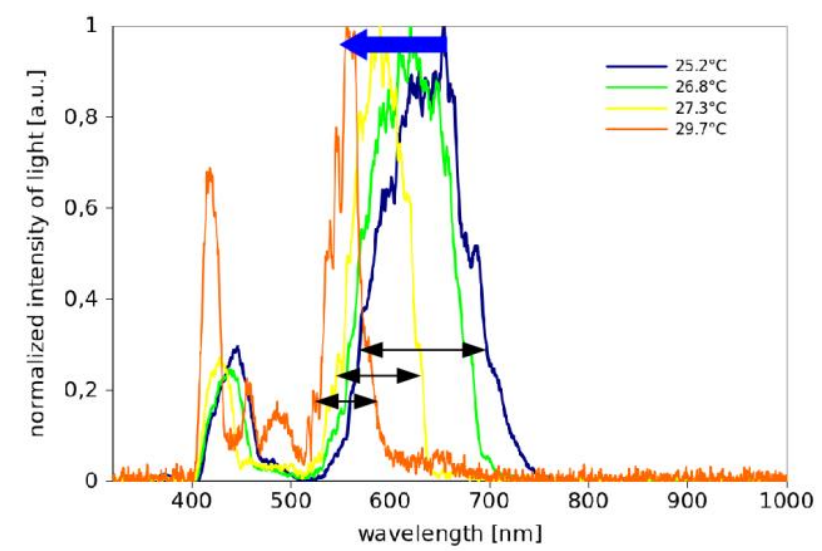

Fig.7. Photonic band gap tuning and narrowing under the influence of temperature.

To conclude, the main advantage of the microstructured PLCFs lies in their tuning possibilities and abilities to modify propagation properties of the PLCFs. In this work the cascade filter based on photonic crystal fiber filled with a low-birefringent liquid crystal mixture was presented. The results show that it is possible to construct a PLCF-based cascade filter with a PBG narrowing effect.
However, photonic crystal fibers with LCs should be appropriately selected to get the same photonic band gaps in the two PLCFs. A successful combination of liquid crystals and photonic crystal fibers technology provides great opportunities for applications in optical devices.

This work is supported by the National Science Centre under the grant no. 2011/01/B/ST7/05015and by the Warsaw University of Technology. One of the authors (M. Tefelska) has been supported by the European Union in the framework of European Social Fund through the Warsaw University of Technology Development Programme, realized by Center for Advanced Studies.

\section{References}

[1] P.St.J. Russell, Science 299, 358 (2003).

[2] C. Knight, Nature 424, 847 (2003).

[3] S. Arismar Cerqueira Jr, Rep. Prog. Phys. 73, 024401 (2010).

[4] T.T. Larsen, A. Bjarklev, D.S. Hermann, J. Broeng, Opt. Exp. 11, 2589 (2003).

[5] P. Roberts, F. Couny, H. Sabert, B. Mangan, D. Williams, L. Farr, M. Mason, A. Tomlinson, T. Birks, J. Knight, and P.St.J. Russell, Opt. Exp. 13(1), 236 (2005).

[6] C. Zhang, G. Kai, Z. Wang, Y. Liu, T. Sun, S. Yuan, X. Dong, Opt. Lett. 30, 2703 (2005).

[7] T.T. Alkeskjold, A. Bjarklev, Opt. Lett. 32, 1707 (2007).

[8] T.R. Woliński, S. Ertman, P. Lesiak, A.W. Domański, A. Czapla, R. Dąbrowski, E. Nowinowski-Kruszelnicki, J. Wójcik, OptoElectr. Rev. 14, 329 (2006).

[9] F. Du, Y.Q. Lu, S.T. Wu, Appl. Phys. Lett. 85, 2181 (2004).

[10] B. Eggleton, Ch. Kerbage, P. Westbrook, R. Windeler, A. Hale, Opt. Exp. 9(13), 698 (2001).

[11] T.R. Woliński, S. Ertman, D. Budaszewski, M. Chychłowski, A. Czapla, R. Dąbrowski, A.W. Domański, P. Mergo, E. Nowinowski-Kruszelnicki, K.A. Rutkowska, M. Sierakowski, M. Tefelska, Proc. SPIE 7955, 795502 (2011).

[12] L. Scolari, T.T. Alkeskjold, A. Bjarklev, Electron. Lett. 42(22), 1270 (2006).

[13] M.M. Tefelska, S. Ertman, T.R. Woliński, R. Dąbrowski, J. Wójcik Phot. Lett. Poland 2(3), 119 (2010).

[14] M.M. Tefelska, M.S. Chychłowski, T.R. Woliński, R. Dąbrowski, J. Wójcik, Phot. Lett. Poland 1(2), 97 (2009).

[15] T.R. Woliński, K. Szaniawska, K. Bondarczuk, P. Lesiak, A.W. Domański， R. Dąbrowski, E. Nowinowski-Kruszelnicki, J. Wójcik, Opto-Electron. Rev. 13(2), 177 (2005).

[16] R. Dąbrowski, J. Dziaduszek, Z. Stolarz, J. Kędzierski, J. Opt. Technol. 72, 662 (2005).

[17] T.R. Woliński, K. Szaniawska, S. Ertman, P. Lesiak, A.W. Domański, R. Dąbrowski, E. Nowinowski-Kruszelnicki, J. Wójcik, Meas. Sci. Technol. 17, 985 (2006). 